\title{
The condensation of (chlorocarbonyl)phenyl ketene with 1,3- dinucleophiles. I. Synthesis of 4-hydroxy-2-(1H)-pyridinones and mesoionic 1,3-oxazinium olates
}

\author{
Mehdi Abaszadeh, Hassan Sheibani,* Kazem Saidi, and Rooholla Moordini Nejad \\ Department of Chemistry, Shahid Bahonar University of Kerman, Kerman 76169, Iran \\ E-mail: $\underline{\text { hsheibani@mail.uk.ac.ir }}$
}

\begin{abstract}
Investigation of the reaction of (chlorocarbonyl)phenyl ketene with 1,3-dinucleophiles such as Schiff bases which were prepared from acetophenone and aryl amides led to the discovery of new synthetic pathways towards 4-hydroxy-3,6-disubstituted-2 $(1 H)$-pyridinones and 2,5-diaryl6-oxo-5-phenyl-6H-1,3-oxazin-3-ium-4-olates respectively. These reactions provide a new route to produce 2-pyridinones and cross - conjugated heterocyclic mesomeric betaines in good to excellent yields in a short reaction time.
\end{abstract}

Keywords: (Chlorocarbonyl)phenyl ketene, 1,3-dinucleophiles, 2(1H)-pyridinones, mesoionic 1,3-oxazinium olates

\section{Introduction}

(Chlorocarbonyl)phenyl ketene 2, first described by Nakanishi, represents a highly reactive equivalent of phenylmalonic acid. ${ }^{1}$ Chlorocarbonyl ketenes (CCKs) are currently of considerable interest, not only because of mechanistic and theoretical considerations, ${ }^{2}$ but also due to their use as synthetic building blocks in the preparation of organic compounds. ${ }^{3}$ These ketenes have been found to be very effective 1,3-dielectrophilic reagents and react with a wide variety of nucleophiles under mild experimental conditions, and have been used mainly for the synthesis of five- and six-membered heterocycles functionalized with oxo and hydroxyl groups in the 1,3positions. $^{4,5}$

Structures containing the $2(1 H)$-pyridinone (2-pyridone) skeleton are rapidly gaining importance in synthetic and natural product chemistry. Compounds incorporating this nucleus are quite versatile as synthetic intermediates. ${ }^{6,7}$ For example, treatment of 2-pyridones with phosphorus pentachloride generates 2-chloropyridines. The pyridone nucleus can be partially reduced via catalytic hydrogenation to piperidinones or further reduced to fully saturated 
piperidines. The diene portion of the molecule can undergo Diels-Alder cycloaddition reactions with dienophiles, or one double bond may act as a dienophile to an added diene. ${ }^{8}$ Some monocyclic pyridones are important medicinally since they exhibit antiinflammatory activity. ${ }^{9}$ Among the naturally occurring substances which incorporate the $2(1 \mathrm{H})$-pyridinone nucleus are ricinine and tenellin. Additional members of this class belong to the Lupinane group of alkaloids, such as cytisine and anagyrine. ${ }^{10}$

The synthesis of 6-chloro-2,3,5-triphenyl-1,3-oxazine-4-one from the reaction of ketene 2 with $N$-benzylideneaniline (Schiff base of benzaldehyde) as a [4+2] cycloaddition product has been reported. ${ }^{11}$ In continuing our previous work on the reaction of chlorocarbonyl ketenes (CCKs) with dinucleophiles, ${ }^{12-16}$ we now wish to describe the condensation of (chlorocarbonyl)phenyl ketene with 1,3-dinucleophiles such as Schiff bases prepared from acetophenone and $N$-substituted benzamides to afford 4-hydroxy-2-(1H)-pyridinone and mesoionic 1,3-oxazinium olate derivatives respectively.

\section{Results and Discussion}

Due to our interest in preparing structures containing the $2(1 H)$-pyridinone skeleton ${ }^{17}$ as exhibited in Scheme 1 the 4-hydroxy-3,6-disubstituted-2(1H)-pyridinone derivatives were prepared in a one step procedure from readily available (chlorocarbonyl)phenyl ketene and Schiff bases such as $N$-phenyl- $N$-[(E)-1-phenylethylidene $]$ amine, $N$-[(E)-1-(4-methylphenyl) ethylidene $]-N$-phenylamine, $N$-benzyl- $N-[-[(E)-1$-phenylethylidene $]$ amine, $N$ - $[(E)$-1-(4-chlorophenyl)ethylidene)](phenyl)methaneamine and $N$ - [(E)-1-(4-chlorophenyl)ethylidene)](4-methylphenyl)methaneamine. This method provides an easy route to prepare 4-hydroxy-3,6-di substituted-2(1H)-pyridinone derivatives in good to excellent yields and a short reaction time (Scheme 1).

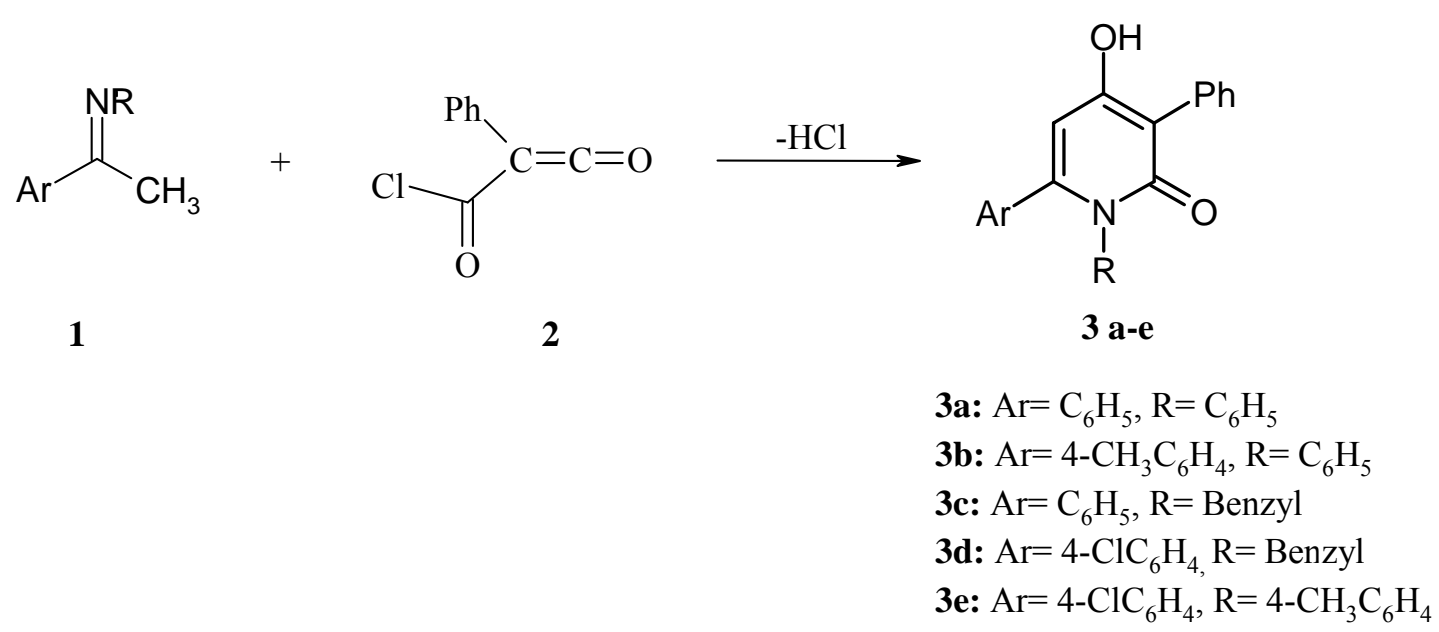

\section{Scheme 1}


We have reported the synthesis of 6-chloro-2,3,5-triphenyl-1,3-oxazine-4-one from the reaction of ketene 2 with $\mathrm{N}$-benzilideneaniline (Schiff base of benzaldehyde) which were refluxed under a nitrogen stream. The product was formed with Schiff bases which are prepared from aryl aldehydes that do not have an $\alpha$-hydrogen. Here we used Schiff bases of acetophenone which contain $\alpha$-hydrogens (Scheme 2).<smiles>O=C=C(C(=O)Cl)c1ccccc1</smiles><smiles>C(=Nc1ccccc1)c1ccccc1</smiles>

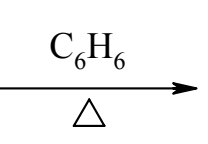<smiles>O=C1C(c2ccccc2)=C(Cl)OC(c2ccccc2)N1c1ccccc1</smiles>

\section{Scheme 2}

There are several reports on the generation of 1,3-oxazinium olates by reaction of chlorocarbonyl ketenes with $\mathrm{N}$-monosubstituted amides in the presence of triethylamine as a base and $\mathrm{HCl}$ scavenger. ${ }^{18,19}$ We now report the preparation of a series of 1,3-oxazinium olates from the reaction of $\mathrm{N}$-monosubsitituted 4-methoxy or 2,4-dimethoxy benzamide derivatives with (chlorocarbonyl)phenyl ketene without the use of triethylamine. The present protocol offers several advantages including high yields, operational simplicity and clean reaction conditions (Scheme 3).<smiles>O=C([AlH2])N[PH3+]</smiles>

4<smiles>O=C=C(C(=O)Cl)c1ccccc1</smiles>

2<smiles>C=C</smiles>

5a: $\mathrm{Ar}=4-\mathrm{MeOC}_{6} \mathrm{H}_{4}$ 5b: $\mathrm{Ar}=2,4-\mathrm{diMeOC}_{6} \mathrm{H}_{3}$

\section{Scheme 3}

It is pertinent to note that (chlorocarbonyl)phenyl ketene undergoes a degenerate 1,3-shift of chlorine, as determined by ${ }^{13} \mathrm{C}-\mathrm{NMR}$ spectroscopy. ${ }^{20}$ The chlorine atom can exchange between two carbonyl groups and also these ketenes can exist as a mixture of two conformers: s-trans and s-cis (Scheme 4). ${ }^{21}$ 


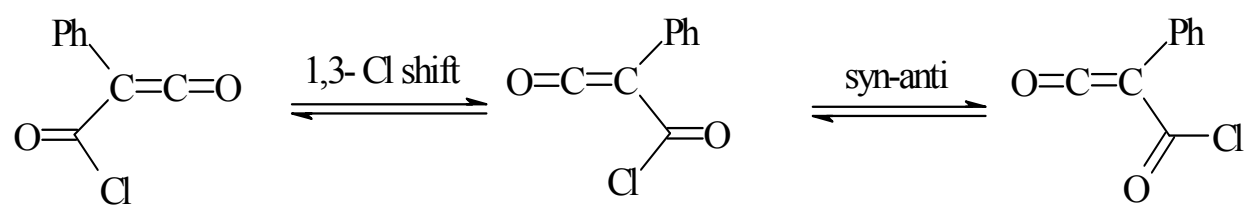

\section{Scheme 4}

The cycloaddition reactions presented in Schemes 1 and 2 were accomplished by boiling equimolar quantities of (chlorocarbonyl)phenyl ketene and the corresponding 1,3-dinucleophiles (Schiff bases and benzamides) in a dry solvent. These compounds are not reactive enough to react with dielectrophiles such as (chlorocarbonyl)phenyl ketene at ambient temperature. However, when they are converted to other tautomeric forms at high temperature, the nitrogen or hydroxy groups of the tautomeric forms will attack the acyl chloride of the ketene followed by ring closure to produce the products. A plausible mechanism for the reaction of (chlorocarbonyl)phenyl ketene with Schiff bases $\mathbf{1}$ is proposed in Scheme 5.<smiles>[R]NC([R])=CC#CC#CCC</smiles><smiles>[R]c1cc(O)c(-c2ccccc2)c(=O)[nH]1</smiles>

\section{Scheme 5}

The structures of compounds 3a-e and 5a, $\mathbf{b}$ were deduced from their elemental analyses and their IR, high-field ${ }^{1} \mathrm{H}$ - and ${ }^{13} \mathrm{C}-\mathrm{NMR}$ spectra. The ${ }^{1} \mathrm{H}-\mathrm{NMR}$ and ${ }^{13} \mathrm{C}$ - NMR spectra of 4hydroxy-3,6-disubstituted-2(1H)-pyridinone derivatives 3a-e exhibited only one tautomer.

\section{Conclusions}

In conclusion we have shown that the condensation reactions of (chlorocarbonyl) phenyl ketene with 1,3-dinucleophiles such as Schiff bases and amides occur efficiently in boiling toluene or xylene, providing a convenient and rapid synthesis of 4-hydroxy-3,6-disubstituted-2(1H)- pyridinone and 1,3-oxazin-3-ium-6-olate derivatives in high yield, by a simple procedure and short 
experimental time. Furthermore the products are solid and precipitate out from the reaction mixture which makes their purification simple.

\section{Experimental Section}

General Procedures. Melting points were measured on a Gallenkamp melting point apparatus and are uncorrected. IR spectra were measured on a Mattson 1000 FT-IR spectrometer. The proton and carbon NMR spectra were recorded with a BRUKER DRX-500 AVANCE spectrometer at 500 and $125.77 \mathrm{MHz}$, respectively. Mass spectra were recorded on a MSQP2000A Shimadzu mass spectrometer operating at an ionization potential of $70 \mathrm{eV}$. Elemental analyses were performed using a Heracus CHN-O-Rapid analyzer.

\section{4-hydroxy-1,3,6-triphenyl-2(1H)-pyridinone (3a). General procedure (3a-e)}

To a stirred solution of $0.39 \mathrm{~g} N$-phenyl- $N-[(E)-1$-phenylethylidene $]$ amine $(2 \mathrm{mmol})$ in $20 \mathrm{~mL}$ dry boiling toluene, a mixture of $0.36 \mathrm{~g}$ (chlorocarbonyl)phenyl ketene $(2 \mathrm{mmol})$ in $5 \mathrm{~mL}$ dry THF was added dropwise over 2 min. The compound 3a was formed immediately as a white precipitate. The reaction mixture was cooled and solid product was collected and recrystallized from dry ethyl acetate. $0.64 \mathrm{~g}$. White crystals, yield 94\%; mp 298-300 ${ }^{\circ} \mathrm{C} ; v_{\max }(\mathrm{KBr}): 3100$ $2850\left(\right.$ broad, s, OH), $1653(\mathrm{C}=\mathrm{O}), 1610,1591(\mathrm{Ar}) \mathrm{cm}^{-1} ; \delta_{\mathrm{H}}\left(500 \mathrm{MHz}, \mathrm{DMSO}-\mathrm{d}_{6}\right): 10.78(1 \mathrm{H}, \mathrm{s}$, $\mathrm{OH}), 7.47-7.15\left(15 \mathrm{H}, \mathrm{m}\right.$, arom), $6.18(1 \mathrm{H}, \mathrm{s}, \mathrm{CH}) \delta_{\mathrm{C}}\left(125 \mathrm{MHz}, \mathrm{DMSO}-\mathrm{d}_{6}\right): 163.57,162.46$, 147.62 , 139.84, 135.27, 134.00, 131.69, 131.55, 130.62, 129.19, 128.79, 128.42, 128.05, 127.07, 123.14, 110.86, 102.57. MS, m/z (\%): 339(M+, 25), 262 (43), 222 (100), 77(79), 51(45). Anal. Calcd. For $\mathrm{C}_{23} \mathrm{H}_{17} \mathrm{NO}_{2}$ : C, 81.40; H, 5.05; N, 4.13 \%. Found : C, 81.27; H. 5.12; N; 4.01 \%.

4-hydroxy-6-(4-methylphenyl)-1,3-diphenyl-2(1H)-pyridinone (3b). A 0.63 g. pale yellow crystals, yield 90\%; mp 287-290 ${ }^{\circ} \mathrm{C}$; $v_{\max }(\mathrm{KBr})$ : 3110-2850 (broad, s, OH), $1634(\mathrm{C}=\mathrm{O}), 1610$ $\left(\right.$ Ar) $\mathrm{cm}^{-1} ; \delta_{\mathrm{H}}\left(500 \mathrm{MHz}, \mathrm{DMSO}-\mathrm{d}_{6}\right): 10.67(1 \mathrm{H}, \mathrm{s}, \mathrm{OH}), 7.45-6.99(14 \mathrm{H}, \mathrm{m}$, arom $), 6.13(1 \mathrm{H}, \mathrm{s}$, $\mathrm{CH}), 2.22\left(3 \mathrm{H}, \mathrm{s}, \mathrm{CH}_{3}\right) ; \delta_{\mathrm{C}}\left(125 \mathrm{MHz}, \mathrm{DMSO}-\mathrm{d}_{6}\right): 163.77,162.54,149.05,138.57,137.52$, 134.98, 133.76, 131.73, 130.98, 130.27, 129.51, 129.40, 129.33, 128.00, 126.94, 110.38, 102.38, 21.46; MS, m/z (\%): 353( $\left.\mathrm{M}^{+}, 100\right), 236$ (78), 135(27), 91 (100), 77(45), 51(25). Anal. Calcd. For $\mathrm{C}_{24} \mathrm{H}_{19} \mathrm{NO}_{2}$ : C, 81.56; H, 5.42; N, $3.96 \%$. Found : C, 81.43; H. 5.12; N; $3.70 \%$.

1-benzyl-4-hydroxy-3,6-diphenyl-2(1H)-pyridinone (3c). A 0.66 g. pale yellow crystals, yield 94\%; mp $281{ }^{\circ} \mathrm{C} ; v_{\max }(\mathrm{KBr}): 3160-2750$ (broad, s, OH), $1649(\mathrm{C}=\mathrm{O}), 1590(\mathrm{Ar}) \mathrm{cm}^{-1} ; \delta_{\mathrm{H}}(500$ $\left.\mathrm{MHz}, \mathrm{DMSO}-\mathrm{d}_{6}\right):$ 10.60(1H, s, OH), 7.43-6.83(15H, m, arom), $5.98(1 \mathrm{H}, \mathrm{s}, \mathrm{CH}), 5.03(2 \mathrm{H}, \mathrm{s}$, $\left.\mathrm{CH}_{2}\right) ; \delta_{\mathrm{C}}\left(125 \mathrm{MHz}, \mathrm{DMSO}-\mathrm{d}_{6}\right)$ : 162.60, 161.14, 148.60, 137.95, 135.13, 134.15, 130.84, 129.22, 128.40, 128.37, 128.21, 127.19, 126.70, 126.21, 126.16, 109.91, 101.69, 47.65; MS, m/z $(\%): 353\left(\mathrm{M}^{+}, 24\right), 352(46), 135(32), 106(26), 91(100), 77(29), 65(24), 51(10)$; Anal. Calcd. For $\mathrm{C}_{24} \mathrm{H}_{19} \mathrm{NO}_{2}$ : C, 81.56; H, 5.42; N, $3.96 \%$. Found : C, 81.53; H. 5.46; N; $3.99 \%$.

1-benzyl-6-(4-chlorophenyl)-4-hydroxy-3-phenyl-2(1H)-pyridinone (3d). A 0.70 g. pale yellow crystals, yield 91\%; mp $296{ }^{\circ} \mathrm{C} ; v_{\max }(\mathrm{KBr}): 3150-2800$ (broad, s, OH), $1651(\mathrm{C}=\mathrm{O})$, 
$1561(\mathrm{Ar}) \mathrm{cm}^{-1} ; \delta_{\mathrm{H}}\left(500 \mathrm{MHz}, \mathrm{DMSO}-\mathrm{d}_{6}\right): 10.61(1 \mathrm{H}, \mathrm{s}, \mathrm{OH}), 7.45-6.85(14 \mathrm{H}, \mathrm{m}$, arom $), 5.98$ $(1 \mathrm{H}, \mathrm{s}, \mathrm{CH}), 5.04\left(2 \mathrm{H}, \mathrm{s}, \mathrm{CH}_{2}\right) ; \delta_{\mathrm{C}}\left(125 \mathrm{MHz}, \mathrm{DMSO}-\mathrm{d}_{6}\right): 162.58,161.06,147.31,137.82$, $134.08,133.90,130.83,130.45,130.31,128.54,128.28,128.02,127.20,126.76,126.18,110.13$, 101.93, 47.58; MS, m/z (\%): 387(M+18), 270(100), 106(26), 91(70), 77(32), 65(47); Anal. Calcd. For $\mathrm{C}_{24} \mathrm{H}_{18} \mathrm{ClNO}_{2}$ : C, 74.32; H, 4.68; N, 3.61 \%. Found : C, 74.26; H. 4.71; N; 3.53 \%.

6-(4-chlorophenyl)-4-hydroxy-1-(4-methylphenyl)-3-phenyl-2(1H)-pyridinone $\quad$ (3e). A 0.66 g. pale yellow crystals, yield $85 \%$; mp $282{ }^{\circ} \mathrm{C} ; v_{\max }(\mathrm{KBr}): 3150-2800$ (broad, s, OH), 1631 $(\mathrm{C}=\mathrm{O}), 1596(\mathrm{Ar}) \mathrm{cm}^{-1} ; \delta_{\mathrm{H}}\left(500 \mathrm{MHz}, \mathrm{DMSO}-\mathrm{d}_{6}\right): 10.79(1 \mathrm{H}, \mathrm{s}, \mathrm{OH}), 7.98-7.03(13 \mathrm{H}, \mathrm{m}$, arom $)$, $6.18(1 \mathrm{H}, \mathrm{s}, \mathrm{CH}), 2.23\left(3 \mathrm{H}, \mathrm{s}, \mathrm{CH}_{3}\right) ; \delta_{\mathrm{C}}\left(125 \mathrm{MHz}, \mathrm{DMSO}-\mathrm{d}_{6}\right): 163.65,162.41,147.71,137.62$, 137.26, 135.41, 133.96, 131.69, 131.52, 130.98, 130.28, 129.72, 128.82, 128.03, 123.93, 110.81, 102.55, 21.46; MS, m/z (\%): 387( $\left.\mathrm{M}^{+}, 65\right), 270(100), 106(35), 91(87)$, 77(39), 65(45); Anal. Calcd. For $\mathrm{C}_{24} \mathrm{H}_{18} \mathrm{ClNO}_{2}$ : C, 74.32; H, 4.68; N, 3.61 \%. Found : C, 74.15; H. 4.34; N; 3.47 \%.

2-(4-methoxyphenyl)-4-oxo-3,5-diphenyl-4H-1,3-oxazin-3-ium-6-olate procedure (5a, b)

(5a). General

A solution of ketene $2(0.36 \mathrm{~g}, 2 \mathrm{mmol})$ in anhydrous $\mathrm{CHCl}_{3}(15 \mathrm{~mL})$ was added dropwise to a stirred solution of 4-methoxy- $N$-phenylbenzamide $(0.45 \mathrm{~g}, 2 \mathrm{mmol})$ in anhydrous $\mathrm{CHCl}_{3}(30 \mathrm{~mL})$ under a nitrogen atmosphere and was refluxed for $2 \mathrm{~h}$. The product $5 \mathrm{a}$ was precipitated from the reaction mixture by cooling, and the solid was filtered and washed with anhydrous diethylether $(50 \mathrm{~mL})$. 0.65 g. pale yellow crystals, yield $85 \%$; mp $273{ }^{\circ} \mathrm{C} ; v_{\max }(\mathrm{KBr}): 1741,1661,1617 \mathrm{~cm}^{-1} ; \delta_{\mathrm{H}}(500 \mathrm{MHz}$, DMSO-d $\left.\mathrm{d}_{6}\right): 7.87-7.00\left(14 \mathrm{H}, \mathrm{m}\right.$, arom), 3.83(3H, s, $\left.\mathrm{CH}_{3}\right) ; \delta_{\mathrm{C}}\left(125 \mathrm{MHz}, \mathrm{DMSO}-\mathrm{d}_{6}\right): 170.87,163.82$, $135.91,131.65,131.39,129.86,129.80,128.96,128.80,128.55,127.82,127.49,126.76,114.80$, 114.54, 109.21, 55.96; MS, m/z (\%): 371( $\left.\mathrm{M}^{+}, 10\right), 357(15), 135(100), 107(60), 91(85), 77(50)$, 39(20); Anal. Calcd. For $\mathrm{C}_{23} \mathrm{H}_{17} \mathrm{NO}_{4}$ : C, 74.38; H, 4.61; N, 3.77 \%. Found : C, 74.06; H. 4.38; $\mathrm{N} ; 3.48 \%$.

2-(2,4-dimethoxyphenyl)-4-oxo-3,5-diphenyl-4H-1,3-oxazin-3-ium-6-olate (5b). A 0.65 g. pale yellow crystals, yield $82 \%$; mp $286{ }^{\circ} \mathrm{C} ; v_{\max }(\mathrm{KBr}): 1766,1655,1617 \mathrm{~cm}^{-1} ; \delta_{\mathrm{H}}(500 \mathrm{MHz}$, DMSO-d $\left.)_{6}\right)$ 7.86-7.20(11H, m, arom), 6.51(1H, s, arom), $6.19(1 \mathrm{H}, \mathrm{s}, \operatorname{arom}), 3.80\left(3 \mathrm{H}, \mathrm{s}, \mathrm{CH}_{3}\right)$, $3.56\left(3 \mathrm{H}, \mathrm{s}, \mathrm{CH}_{3}\right) ; \delta_{\mathrm{C}}\left(125 \mathrm{MHz}, \mathrm{DMSO}-\mathrm{d}_{6}\right): 169.53,165.42,157.62,157.42,157.17,134.73$, $132.55,131.68,129.74,129.43,129.02,128.85,128.73,128.16,127.62,126.07,105.55,98.19$, 55.63, 55.18; MS, m/z (\%): 401( $\left.\mathrm{M}^{+}, 5\right), 253(33), 165(100), 129(29), 91(54), 65(52), 51(25)$, 39(42); Anal. Calcd. For $\mathrm{C}_{24} \mathrm{H}_{19} \mathrm{NO}_{5}$ : C, 71.81; H, 4.77; N, 3.49 \%. Found : C, 71.59; H. 4.61; $\mathrm{N} ; 3.53 \%$.

\section{Acknowledgements}

The authors express appreciation to the Shahid Bahonar University of Kerman Faculty Research Committee for its support of this investigation. 


\section{References and Notes}

1. Nakanishi, S.; Butler, K. Org. Prep. Precd. Int. 1975, 7(4), 155.

2. Wallfisch, B. C.; Belaj, F.; Wentrup, C.; Kappe, C. O.; Kollenz, G. J. Chem. Soc, Perkin Trans 1 2002, 599.

3. Brown, D. G.; Hoye, T, R.; Brisbois, R. G. J. Org. Chem. 1998, 63, 1630.

4. Ried, W.; Nenninger, H. Synthesis 1990, 167.

5. Potts, K. T.; Dery, M. O.; Kullnig, R. J. Chem. Soc., Chem. Commun. 1987, 840.

6. Earl, R. A.; Vollhardt, K. P. C. J. Org. Chem. 1984, 49, 4786.

7. Katritzky, A. R.; Silina, A.; Tymoshenko, D. O.; Qiu, G.; Nair, S.K.; Steel, P.J. Arkivoc 2001, vii, 138.

8. Tieckelmann, H. In 'Pyridine and its Derivatives"; Abramovitch, R. A., Ed.; Interscience: New York, 1974; Vol. 14 Supplement, Part 3, Ch. 12, p. 597.

9. Pierce, J. B.; Ariyan, 2. S.; Ovenden, G. S. J. Med. Chem. 1982, 25, 131.

10. (a) Pelletier, S. W. "Chemistry of the Alkaloids"; Van Nostrand-Reinhold: New York, 1970. 'The Alkaloids". (b) Specialist Reports; The Royal Society of Chemistry: London, 19691982; Vol. 1- 12. (c) "The Alkaloids"; Academic Press: New York, 1950-1983; Vol. 1-21.

11. Saidi, K.; Shaterian, H.; Aghaei, D. Heterocycl. Commun. 2000, 6, 93.

12. Sheibani, H.; Islami, M. R.; Hojatollah Khabazzadeh; Saidi, K. Tetrahedron 2004, 60, 5932.

13. Sheibani, H.; Mosslemin, M. H.; Behzadi, S.; Islami,M. R.; Foroughi, H.; Saidi, K. Arkivoc 2005, $x v, 88$.

14. Sheibani,H.; Mosslemin, M. H.; Behzadi, S.; Islami, M. R.; Saidi, K. Synthesis 2006, 3, 435.

15. Sheibani, H.; Bernhardt, P.V.; Wentrup, C. J. Org. Chem. 2005, 70, 5859.

16. George, L.; Veedu, R. N.; Sheibani, H.; Taherpour, A. A.; Flammang, R.; Wentrup, C. J. Org. Chem. 2007, 72, 1399.

17. Sheibani, H.; Saljoogi, A. S.; Bazgir, A. Arkivoc 2008, ii, 115.

18. Potts, K. T.; Dery, M. O.; Kullnig, R. K. J. Chem. Soc. Commun. 1987, 840.

19. Ollis, W. D.; Stanforth, S. P.; Ramsden, C. Tetrahedron 1985, 41, 2239.

20. Finnerty, J.; Andraos, J.; Wong, M. W.; Yamamoto, Y.; Wentrup, C. J. Am. Chem. Soc. 1998, 120, 1701.

21. Koch, R.; Wong, M.W.; Wentrup, C. J. Org. Chem. 1996, 61, 6809. 